\title{
ESTUDO SOBRE A INCIDÊNCIA DE PARTOS NA ADOLESCÊNCIA EM UM MUNICÍPIO DO ESTADO DE SÃO PAULO
}

\author{
Maria Isabel Ruiz Beretta * \\ Fátima Elisabeth Denari ** \\ João Carlos Pedrazzani ***
}

Este trabalho apresenta a incidência de partos na adolescência, ocorridos no município de São Carlos-SP, durante um ano ( $2^{\circ}$ semestre de 1993 e $1^{\circ}$ semestre de 1994). Encontrou-se um percentual de $14,72 \%$ de ocorrência de partos na faixa etária de 11 a 18 anos. Além dessa incidência, também verificou-se o número de gestações, ocupação, tipo de parto e estado civil. Tais fatores poderiam configurar a necessidade de programas educacionais específicos para esta clientela.

UNITERMOS: parto, adolescência, São Carlos (SP)

\section{INTRODUÇÃO}

A partir do momento em que a gravidez na adolescência é entendida como problemática, torna-se necessário justificar o porque e de que forma essa questão constitui-se em objeto de pesquisa.

\footnotetext{
* Professor Auxiliar do Departamento de Enfermagem da Universidade Federal de São Carlos

** Professor Assistente do Departamento de Psicologia da Universidade Federal de São Carlos

*** Professor Adjunto do Departamento de Enfermagem da Universidade Federal de São Carlos
} 
Em 1974, na "Reunião sobre gravidez e aborto na adolescência" patrocinada pela Organização Mundial de Saúde (OMS), a adolescência foi definida como sendo o período da vida em que: o indivíduo passa do aparecimento das características sexuais secundárias á maturidade sexual; os padrões psicológicos e a identificação do indivíduo evoluem da fase infantil para a fase adulta; ocorre a transição do estado de total dependência sócio-econômica para o de relativa independência.

Em algumas sociedades, como apontam BECKER (1989) e MONROY de VELASCO (1988), a transição do status infantil para o de adulto se faz em função do critério biológico, a puberdade simbolizando a condição de estar em condição adulta e, portanto, apto para assumir novas responsabilidades, entre elas, o casamento. Em outras, tal transição se faz paulatinamente, sem que haja o reconhecimento social de uma fase intermediária entre a infância e a idade adulta.

Nas sociedades industrializadas, em que se fazem rigorosos os critérios de maturidade intelectual e capacitação técnica, a adolescência é entendida como um período em que ocorre o preparo do jovem para o ingresso, com êxito, no mundo dos adultos (OMS, 1975).

A adolescência não deve ser reconhecida apenas como fenômeno biológico, mas sim como um período com caráter social e histórico, assumindo uma conotação diferente dentro do contexto onde o jovem se encontra.

Ainda tentando delimitar um período cronológico para o início e término da adolescência, alguns autores apontam, respectivamente, as faixas etárias de 11-12 e 19-21 anos (GAUDERER, 1986; DOLTO, 1990; VASCONCELOS, 1992).

Embora a literatura aponte para discrepâncias no que se refere à cronologia, alguns pontos parecem ser comuns a estudiosos e autores que trabalham com adolescentes. Dentre eles, podem-se citar a curiosidade, a inquietude, o dilema de não estar mais criança e não ainda adulto, as tarefas do desenvolvimento, a questão da afirmação e, principalmente, as descobertas.

Segundo DOLTO (1990), os "rituais de passagem" constituem uma das mais importantes tarefas do desenvolvimento e caracterizam uma das mais polêmicas descobertas: a sexualidade. $\mathrm{E}$ a explosão que tal descoberta implica pode resultar em condições que, certamente, desencadeiam problemas.

Com relação à gravidez na adolescência, considera-se ser esta um problema de âmbito social por implicar, simultaneamente, em dois fenômenos do desenvolvimento humano: a adolescência e a gestação, além das repercussões biológicas e psicológicas que ela acarreta.

A gravidez em qualquer época é uma situação que sempre gera alterações: mudanças da mulher no seu papel social, reajustamentos interpessoais e intrapsíquicos e, quando esta ocorre na adolescência, as alterações assumem um risco maior, pois, é um período de vida em que há uma superposição de crises vitais: a de um organismo infantil para um organismo adulto com reflexos somáticos e psíquicos.

$\mathrm{Na}$ América Latina, 3.312.000 crianças nascem a cada ano de mães adolescentes. Mundialmente, de cada 100 adolescentes entre 15 e 19 anos, cinco se convertem em mães anualmente, o que ascende a um total de 22.473 .600 crianças nascidas de pais adolescentes por ano (ORGANIZACIÓN PANAMERICANA DE 
LA SALUD - OPS/OMS 1992). A incidência de gravidez em adolescentes no Brasil é de $10 \%$, sendo o número total de meninas adolescentes 28 milhões, ou seja, 2,8 milhões de adolescentes brasileiras entre 10 e 19 anos têm probabilidade de engravidar (IBGE, 1992).

As adolescentes grávidas formam um grupo de risco tanto para elas mesmas como para seus filhos: (ainda que a situação biológica não seja um presente risco) o risco é social e psicológico, já que a gravidez na adolescência ocorre na maioria das vezes entre jovens com limitados recursos e com acesso restrito aos serviços de saúde (OPS/OMS, 1992).

SILVA (1981), salienta o fato de que também como resultado da maternidade precoce, a jovem tem restringidas suas opções educacionais e profissionais, contribuindo desta forma para a manutenção de um estado sócio-econômico carente.

SILVA \& SARMENTO (1988), consideram a maternidade na adolescência como problemática do ponto de vista biopsicossocial e também acreditam que a adolescente não esteja apta, emocional e biologicamente para enfrentar a gravidez, o parto e o processo de criação de um filho, acontecendo geralmente de uma forma inesperada. Isso pode acarretar uma série de episódios negativos que vão interferir no desenvolvimento da jovem, tais como, rejeição familiar e restrições sociais e econômicas.

SANTOS et al (1987), afirmam que a problemática da gravidez na adolescência assume amplitude mundial e sugerem a realização de estudos regionalizados para a elaboração de programas de saúde que atendam as reais necessidades desta clientela.

Desta forma, a questão da gravidez em adolescentes, seja por falta de orientação, informação ou de acesso aos meios contraceptivos, seja pelo contexto em que se encontra, é um problema que afeta a sociedade de uma forma geral, acarretando uma diminuição da possibilidade de melhoria da situação sócioeconômica das jovens e reduzindo sensivelmente as opções educacionais e profissionais.

Em nossa realidade com base em muitos relatos de profissionais ligados à área de saúde, notou-se que a gestação em adolescentes pode estar alcançando índices significativos que denotam a necessidade de um atendimento específico para esta clientela.

A observação desta realidade pelos profissionais da Saúde demonstra a necessidade da implantação de programas de educação em Saúde específicos para adolescentes, o que poderia contribuir para o exercício das atividades das disciplinas da área materno-infantil do Departamento de Enfermagem da Universidade Federal de São Carlos.

Considerando a importância do tema, é que os autores desta pesquisa se propuseram a identificar a incidência de partos na adolescência, na faixa etária de 11 a 18 anos, numa das maternidades do município de São Carlos-SP, abordando alguns aspectos bio-psicossociais com vistas à proposição futura de um programa de Educação em Saúde. 


\section{PROCEDIMENTOS}

Os dados relativos a esta pesquisa foram obtidos a partir das fichas de internação da Maternidade Francisca Cintra Silva da Irmandade da Santa Casa de Misericórdia de São Carlos e, dos registros do Cartório de Registro Civil São Carlos, localizados no município de São Carlos.

Foram pesquisados todos os registros de partos e nascimentos ocorridos no período de 16 de julho de 1993 a 16 de julho de 1994, num total de 3.196 nascimentos neste município. $\mathrm{Na}$ maternidade em que foi desenvolvido o presente estudo a população correspondeu a um registro de 2.392 partos.

Empregou-se a análise estatística na apuração da freqüência e do percentual dos aspectos estudados. Esses aspectos referem-se à incidência da gestação e primi e multiparidade na adolescência, tipo de parto, estado civil e ocupação e, estão dispostos em gráficos e tabelas. O critério para a escolha destes aspectos deveu-se ao fato destes serem freqüentes na literatura e possibilitarem o surgimento de dados comprobatórios de algumas condições. Alguns dados referentes às gestantes adolescentes foram comparados com a população acima de 18 anos atendida na maternidade em estudo.

\section{RESULTADOS E DISCUSSÃO}

Far-se-á a apresentação e também discussão dos resultados encontrados que foram submetidos à análise e demonstrados através de tabelas de freqüência simples e gráficos.

Foram analisados 2.392 registros de partos da maternidade em estudo, dos quais $352(14,72 \%)$ foram de adolescentes de 11 a 18 anos, conforme se observa no Gráfico 1.

\section{GRÁFICO 1 - NÚMERO E PERCENTUAL DE PARTOS NA ADOLESCÊNCIA EM SÃO CARLOS-SP, 1993/1994}

\section{Adolescentes 352}

$(14,72 \%)$

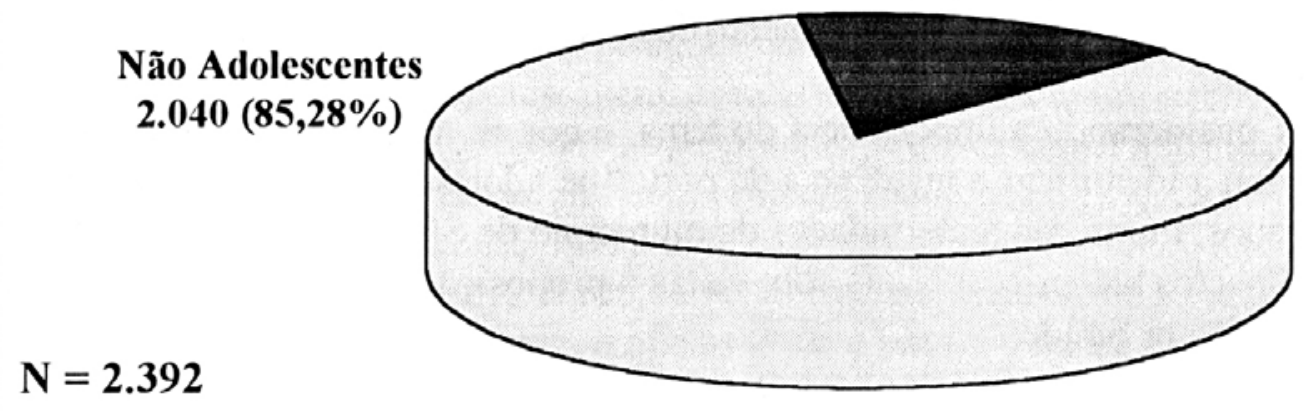


A incidência de partos na adolescência neste estudo, mostra-se superior à encontrada por VITIELLO et al (1982) na cidade de Santo André-SP e, LUZ et al (1983) na cidade de Porto Alegre-RS, que referem 12,3\%e 12,39\% respectivamente; e mostra-se inferior à encontrada por PAIVA et al (1992) na cidade de São Paulo-SP que referem $25,07 \%$.

$\mathrm{Na}$ Tabela 1 analisa-se o estado civil das adolescentes estudadas levando-se em conta sua idade.

TABELA 1 - DISTRIBUIÇÃO DAS ADOLESCENTES ESTUDADAS SEGUNDO 0 ESTADO CIVIL. SÃO CARLOS-SP, 1993/1994

\begin{tabular}{|c|c|c|c|c|}
\hline \multirow{2}{*}{ Idade } & \multicolumn{4}{|c|}{ Estado Civil } \\
\cline { 2 - 5 } & \multicolumn{2}{|c|}{ Solteira } & \multicolumn{2}{c|}{ Casada } \\
\cline { 2 - 5 } 11 & $\mathrm{~N}^{\circ}$ & $\%$ & - & $\mathrm{N}^{\circ}$ \\
12 & 1 & 0,28 & - & - \\
13 & 2 & 0,56 & - & - \\
14 & 4 & 1,14 & 2 & 0,56 \\
15 & 20 & 5,59 & 6 & 1,71 \\
16 & 22 & 6,27 & 19 & 5,39 \\
17 & 47 & 13,38 & 42 & 11,95 \\
18 & 69 & 19,62 & 26 & 7,40 \\
\hline Total & 92 & 26,15 & 95 & 27,01 \\
\hline
\end{tabular}

Ao analisar os resultados apresentados na Tabela I, constata-se que a maior parte das adolescentes 257 (72,99\%) são solteiras e, apenas 95 (27,01\%) são casadas. Esses resultados se assemelham aos apresentados por VITIELLO et al (1983) e, MATHIAS et al (1985), com os correspondentes percentuais de $55 \%$ de solteiras e $45 \%$ de casadas e, $92,3 \%$ de solteiras e $7,7 \%$ de casadas.

Pode-se observar também que em todas as idades aqui relacionadas, o estado civil predominante é solteira.

Quanto à ocupação das adolescentes, a sua apresentação encontra-se a seguir na Tabela 2. Através desta, pode-se observar a grande incidência $(92,96 \%)$ de jovens que se dedicam somente a atividades relativas ao lar. 
TABELA 2 - DISTRIBUIÇÃO DAS OCUPAÇÕES DAS ADOLESCENTES ESTUDADAS EM SÃO CARLOS-SP, 1993/1994

\begin{tabular}{|c|c|c|c|c|c|c|c|c|}
\hline \multirow{2}{*}{ Idade } & \multicolumn{9}{|c|}{ Ocupação } \\
\cline { 2 - 9 } & \multicolumn{2}{|c|}{ Do Lar } & \multicolumn{2}{c|}{ Estudante } & \multicolumn{2}{c|}{ Emp. Dom. } & \multicolumn{2}{c|}{ Serv. Gerais } \\
\cline { 2 - 9 } & $N^{\circ}$ & $\%$ & $N^{\circ}$ & $\%$ & $N^{\circ}$ & $\%$ & $N^{\circ}$ & $\%$ \\
\hline 11 & 1 & 0,28 & - & - & - & - & - & - \\
\hline 12 & 2 & 0,56 & - & - & - & - & - & - \\
\hline 13 & 4 & 1,13 & - & - & - & - & - & - \\
\hline 14 & 22 & 6,26 & - & - & - & - & - & - \\
\hline 15 & 27 & 7,70 & 1 & 0,28 & - & - & - & - \\
\hline 16 & 59 & 16,79 & 3 & 0,84 & - & - & 4 & 1,13 \\
\hline 17 & 106 & 30,12 & 2 & 0,56 & 1 & 0,28 & 2 & 0,56 \\
\hline 18 & 106 & 30,12 & 3 & 0,84 & 2 & 0,56 & 7 & 1,99 \\
\hline Total & 327 & 92,96 & 9 & 2,52 & 3 & 0,84 & 13 & 3,68 \\
\hline
\end{tabular}

Emp. Dom. = empregada doméstica

Serv. Gerais = (balconista, auxiliares de dentista, empacotadoras de supermercado)

Nota-se que apenas 16 (4,52\%) trabalham, distribuindo-se em: $3(0,84 \%)$ empregadas domésticas e $13(3,68 \%)$ em serviços gerais. Com relação às estudantes, pode-se observar um número pequeno de adolescentes que freqüentam escolas, já que apenas 9 (2,52\%) referem estudar. Quanto à idade e número de gestações das adolescentes, pode-se observar a apresentação dos dados na Tabela 3.

TABELA 3 - DISTRIBUIÇÃO DAS OCORRÊNCIAS DE GESTAÇÃO DAS ADOLESCENTES ESTUDADAS EM SÃO CARLOS-SP, 1993/1994

\begin{tabular}{|c|c|c|c|c|c|c|c|c|c|c|}
\hline \multirow{3}{*}{ Idade } & \multicolumn{8}{|c|}{ No de Gestações } & & \\
\hline & \multicolumn{2}{|c|}{ I } & \multicolumn{2}{|c|}{ II } & \multicolumn{2}{|c|}{ III } & \multicolumn{2}{|c|}{ IV } & \multicolumn{2}{|c|}{ Total } \\
\hline & $\mathrm{N}^{0}$ & $\%$ & $\mathrm{~N}^{\mathrm{O}}$ & $\%$ & $\mathrm{~N}^{\circ}$ & $\%$ & $\mathrm{~N}^{\mathrm{O}}$ & $\%$ & $\mathrm{~N}^{\mathrm{O}}$ & $\%$ \\
\hline 11 & 1 & 0,28 & - & - & - & - & - & - & 1 & 0,28 \\
\hline 12 & 2 & 0,56 & - & - & - & - & - & - & 2 & 0,56 \\
\hline 13 & 3 & 1,14 & - & - & - & - & - & - & 4 & 1,14 \\
\hline 14 & 22 & 6,26 & - & - & - & - & - & - & 22 & 6,26 \\
\hline 15 & 28 & 7,95 & - & - & - & - & - & - & 28 & 7,95 \\
\hline 16 & 59 & 16,76 & 6 & 1,71 & 1 & 0,28 & - & - & 66 & 18,75 \\
\hline 17 & 92 & 26,14 & 19 & 5,39 & - & - & - & - & 111 & 31,53 \\
\hline 18 & 91 & 25,85 & 22 & 6,26 & 4 & 1,14 & 1 & 0,28 & 118 & 33,53 \\
\hline Total & 299 & 84,94 & 47 & 13,36 & 5 & 1,42 & 1 & 0,28 & 352 & 100 \\
\hline
\end{tabular}


Ao analisar os resultados apresentados na tabela 3, constata-se que a maioria das adolescentes 299 (84,94\%) eram primigestas. Em contrapartida, encontrou-se adolescente com 18 anos que já era quartigesta. PAIVA et al (1992), também referem ter encontrado em seu estudo, gestantes que aos 16 anos já estavam na terceira gestação. Gestações sucessivas ocasionam transtornos físicos, emocionais e até sociais e, no caso da adolescente, com maior intensidade (SILVA, 1981; GAUDERER, 1986).

A seguir, apresentam-se os resultados relativos aos tipos de parto encontrados através deste estudo, sendo destacados nos Gráficos 2 e 3 e na Tabela 4.

\section{GRÁFICO 2 - NÚMERO E PERCENTUAL DOS TIPOS DE PARTOS OCORRIDOS NA POPULAÇÃO GERAL DA MATERNIDADE EM ESTUDO DE SÃO CARLOS-SP, 1993/1994}

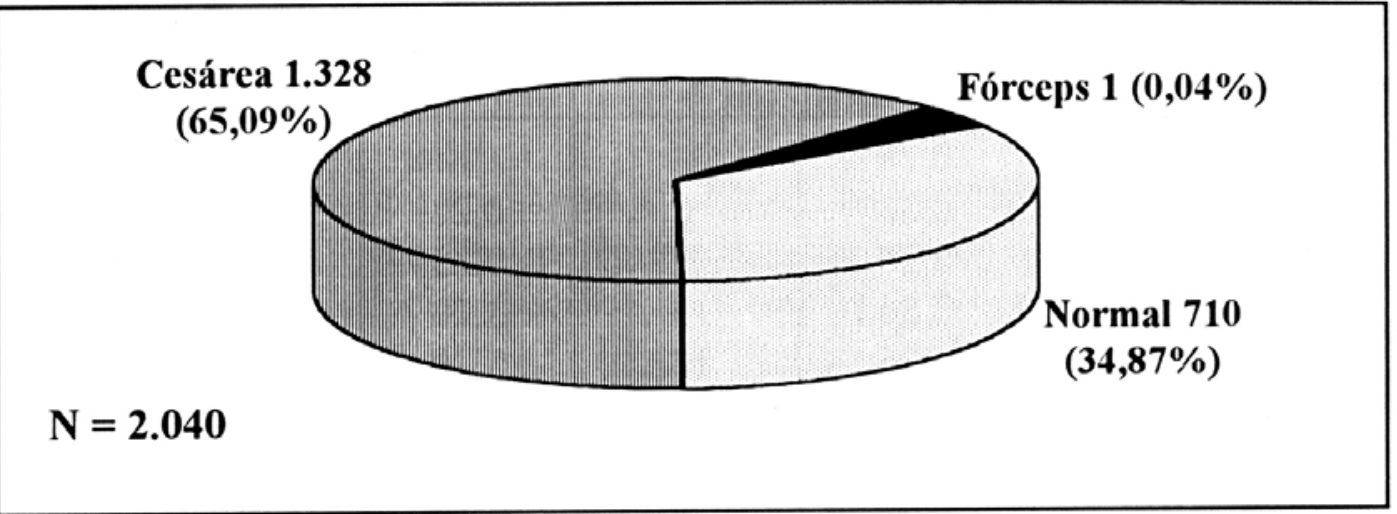

Pode-se observar que um total de $65,09 \%$ das resoluções obstétricas da população geral atendida na maternidade em que se realizou o presente estudo foi a cesárea, seguida por $34,87 \%$ de parto normal e $0,04 \%$ de fórceps.

Constata-se também através do Gráfico 3, a predominância da cesárea também com relação às resoluções obstétricas das adolescentes.

\section{GRÁFICO 3 - NÚMERO E PERCENTUAL DOS TIPOS DE PARTOS OCORRIDOS EM ADOLESCENTES DA MATERNIDADE EM ESTUDO DE SÃO CARLOS-SP, 1993/1994}

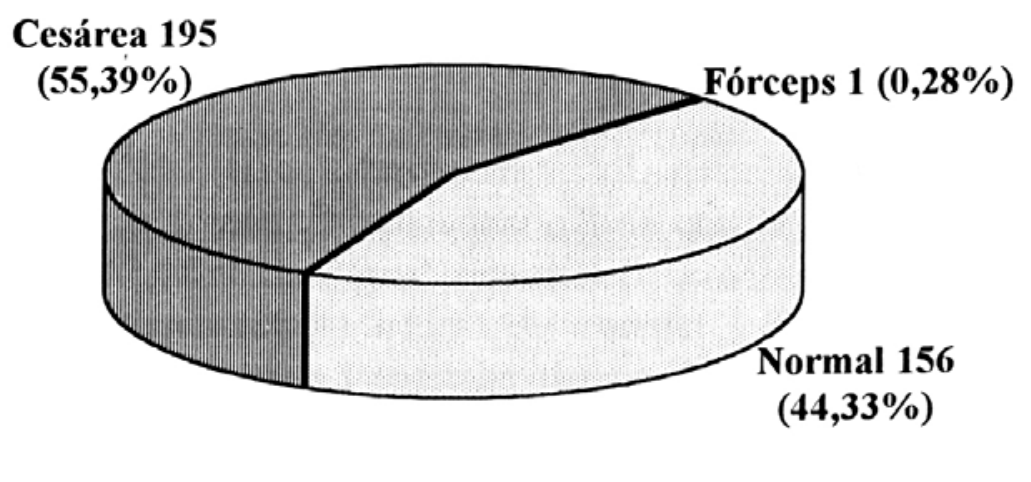

$\mathrm{N}=352$ 
Os dados do Gráfico 3 apresentam um percentual de 55,39\% para cesárea, $44,33 \%$ para parto normal e $0,28 \%$ para fórceps.

Observa-se que, embora haja diferenças nas faixas etárias das duas populações, as resoluções obstétricas são semelhantes.

Os dados da Tabela 4 apresentam com mais detalhes os tipo de partos em adolescentes, relacionando-os com a idade.

TABELA 4 - DISTRIBUIÇÃO DOS TIPOS DE PARTOS OCORRIDOS EM ADOLESCENTES EM SÃO CARLOS-SP, 1993/1994

\begin{tabular}{|c|c|c|c|c|c|c|}
\hline \multirow{3}{*}{ Idade } & \multicolumn{4}{|c|}{ Tipo de Parto } & & \\
\hline & \multicolumn{2}{|c|}{ Parto Normal } & \multicolumn{2}{|c|}{ Cesárea } & \multicolumn{2}{|c|}{ Total } \\
\hline & $\mathrm{N}^{0}$ & $\%$ & $\mathrm{~N}^{\circ}$ & $\%$ & $\mathrm{~N}^{\circ}$ & $\%$ \\
\hline 11 & 1 & 0,28 & - & - & 1 & 0,28 \\
\hline 12 & 1 & 0,28 & 1 & 0,28 & 2 & 0,56 \\
\hline 13 & 1 & 0,28 & 3 & 0,85 & 4 & 1,13 \\
\hline 14 & 9 & 2,56 & 13 & 3,70 & 22 & 6,26 \\
\hline 15 & 15 & 4,26 & 13 & 3,70 & 28 & 7,96 \\
\hline 16 & 26 & 7,40 & 39 & 11,12 & 65 & 18,51 \\
\hline 17 & 55 & 15,61 & 56 & 15,96 & 111 & 31,62 \\
\hline 18 & 48 & 13,66 & 70 & 19,78 & 118 & 33,61 \\
\hline Total & 156 & 44,33 & 195 & 55,39 & 351 & 100 \\
\hline
\end{tabular}

Conforme os dados apresentados na tabela 4, observa-se que, exceto na idade de 11 anos, para todas as idades, o tipo de resolução obstétrica não foi somente parto normal. A literatura (REZENDE, 1974; DELASCIO \& GUARIENTO, 1981) aponta como principais indicações para cesárea os seguintes fatores: sofrimento fetal, desproporção céfalo-pélvica e cesárea anterior. Toma-se difícil inferir algum aspecto que explique a incidência encontrada, pelo fato de que as indicações de cirurgia são variadas e nem sempre concordantes, com a literatura ficando a critério de cada obstetra.

Incidências diferentes foram encontradas por BELEZA et al (1984), em um estudo realizado na cidade de Florianópolis-SC, com 130 parturientes em que constataram para o grupo de adolescentes, freqüência de $81,54 \%$ para parto normal e 18,46\% para cesárea e, também MADI et al (1986), em um estudo realizado na cidade de Caxias do Sul-RS, com 46 adolescentes, constataram uma freqüência de 71,74\% para parto normal, 23,91\% para cesárea e 4,35\% para fórceps. 


\section{CONCLUSÃO}

Como já referenciado na introdução, os profissionais de saúde vem observando um aumento na incidência da gestação na adolescência, no município de São Carlos. Neste estudo encontrou -se um percentual de 14,72 \%, o que gera a necessidade de dar seguimento a estudos desta natureza.

O grande número de mães adolescentes solteiras, sem atividades sócioeconômicas rentáveis, com gestações sucessivas, demonstra a problemática social enfrentada pelas jovens e pela sociedade impondo emergentes soluções assistenciais e educacionais para a saúde. Tais soluções poderiam advir através de programas que forneçam através de discussão em grupo, orientações sobre anatomia e fisiologia do corpo humano, utilização de métodos anticoncepcionais e importância de um planejamento familiar prevenindo a gravidez indesejada, a multiparidade e o abortamento ilegal.

As resoluções obstétricas para as adolescentes, revelaram-se semelhantes às adotadas para a população geral, porém acredita-se que a população de adolescentes deveria ter uma assistência específica, com rotinas de procedimentos adequados para a faixa etária.

A assistência e à orientação deveriam ser iniciadas na pré adolescência, antes que acontecesse a gravidez, sendo realizada em serviços especializados e com equipe especializada, da qual fariam parte vários profissionais tais como médicos, enfermeiras obstetras, psicólogos, pedagogos e assistentes sociais, que poderiam estender esse atendimento às escolas, igrejas e centros comunitários que congregam um grande contingente de adolescentes.

\section{STUDY ON THE RA TE OF CHILDBIRTH BY TEENAGER MOTHERS IN A MUNICIPALITY OF THE STATE OF SÃO PAULO}

The main goal of this study is to find out the rate of child birth by teenager mothers in São Carlos-SP from July, 1993 to July, 1994. The data were collected during one year and showed that in 14, 72\% of the birth the mothers were 11 to 18 years old. The data were also analyzed in terms of, number of pregnancies, occupation, type of birth, and the mother's marital status. The results are discussed in terms of their implication with regard to educational programs to adolescents.

UNITERMS: childbirth, adolescence, São Carlos (SP) 


\title{
ESTUDIO SOBRE LA INCIDENCIA DE PARTOS EN LA ADOLESCENCIA, EN UN MUNICIPIO DEL ESTADO DE SÃO PAULO
}

\begin{abstract}
Este trabajo presenta la incidencia de partos en la adolescencia, ocurridos en el municipio de São Carlos (São Paulo), durante un año (II semestre de 1993 y I semestre de 1994). Se encontró un porcentaje de 14,72\% de ocurrencia de partos en la franja etárea de 11 a 18 años. Además de esa incidencia, también se verificó el número de gestaciones, ocupación, tipo de parto y estado civil. Tales factores configuran la necesidad de programas educacionales específicos para esta clientela.
\end{abstract}

UNITERMOS: parto, adolescencia, São Carlos (SP)

\section{REFERÊNCIAS BIBLIOGRÁFICAS}

01. BECKER, D. O que é adolescência. 7. ed. São Paulo: Brasiliense, 1989. (Coleção Primeiros Passos, n. 159).

02 BELEZA, F. A. et al. Gestação na adolescência. J.Bras.Ginecol., Rio de Janeiro, v. 94, p. 271-74, 1984.

03. DELASCIO, D., GUARIENTO, A. Obstetrícia normal Briquet. São Paulo: Sarvier, 1981.

04. DOLTO, F. A causa dos adolescentes. Rio de Janeiro: Nova Fronteira, 1990.

05. FUNDAÇÃO IBGE. Anuário estatístico do Brasil. Rio de Janeiro, 1992.

06. GAUDERER, E. C. Adolescência, os jovens e nós: uma visão pessoal. J. Pediatr., Rio de Janeiro, v. 61, n. 2, p. 132-50, 1986.

07. LUZ, A.M.H., MENDES, S. M. A., AGOSTINI, S. M. M. Gravidez na adolescência: atuação da enfermeira. Rev .Bras.Enfermagem, Brasília, v. 36, n. 1, p. 3-12, jan/mar 1983.

08. MATHIAS, L. et al. Gravidez na adolescência. IV: Idade limite de risco reprodutivo entre adolescentes. J.Bras.Ginecol., Rio de Janeiro, v. 95, n. 4, p. 141-43, 1985.

09. MADI, J. M., CHIARADIA, A., LUNARDI, P. V. Gravidez na adolescência: a propósito de 46 casos. J.Bras.Ginecol., Rio de Janeiro, v. 96, n. 6, p. 267-70, 1986.

10. MONROY DE VELASCO, A. Fecundidad en la adolescencia: causas, riesgos y opciones. Washington, D. C., Organização Panamericana de la Salud, 1988. (Cuad.tec. n. 12) 
11. ORGANIZACIÓN MUNDIAL DE LA SALUD. EI embarazo y el aborto en la adolescencia. Ginebra: OMS, 1975. (Série de Informes Técnicos 583).

12. ORGANIZACIÓN PANAMERICANA DE LA SALUD OPS/OMS. Salud reproductiva en las Américas, 1992.

13. PAIVA, M. S., MARIN, H.F., MIYAZAWA, N. S. Gestação na adolescência. Rev.Baiana Enfermagem, Salvador, v. 5, n. 1, p. 66-78, out. 1992.

14. REZENDE, J. de. Obstetrícia. 3. ed. Rio de Janeiro: Guanabara Koogan, 1974. 15. SANTOS, E. S. et al. Maternidade e adolescência: sentimentos e atitudes. Rev.Gaúch.Enfermagem, Porto Alegre, v. 8, n. 1, p. 17-26, jan. 1987.

16. SILVA, J.L.P. Fertilidade na adolescência. J.Bras.Ginecol., Rio de Janeiro, v. 91, n. 2, p. 119-23,1981.

17. SILVA, J.L.P., SARMENTO, R.C. Gravidez adolescência e saúde. São Paulo. Comissão de Saúde do Adolescente. Paris Editorial/Secretaria de Estado da Saúde, 1988.

18. VASCONCELOS, N. de. Amor e sexo na adolescência. São Paulo: Moderna, 1985. 